\title{
APPARENT SUPERLUMINOUS QUASI-SOLITONS IN STIMULATED BRILLOUIN BACKSCATTERING
}

\author{
O. LEGRAND* ** and C. MONTES ${ }^{*} * *$ \\ * Laboratoire de Physique de la Matière Condensée, Université de Nice, \\ * ${ }^{*}$ Parc Valrose. F-06034 Nice Cedex, France \\ observatoire de Nice, BP. 139, F-06003 Nice Cedex, France
}

\begin{abstract}
The process of stimulated Brillouin backscattering (SBS) in nonlinear optics is investigated through the well known 3-wave resonant interaction model. The great variety of transient behaviors are schematically distributed among two main classes of asymptotics. Arguments are given in favor of the physical significance of a superluminous backscattered envelope of the solitary wave type and suggestions are made for its observation in an SBS-fiber-ring laser experiment.
\end{abstract}

\section{Résumé}

Le mécanisme de rétrodiffusion Brillouin stimulée (SBS) en optique non linéaire est étudié à l'aide du modèle bien connu de l'interaction résonnante à 3 ondes. La diversité des comportements transitoires est schématiquement classée en deux types principaux d'asymptotiques. Des arguments sont avancés en faveur du sens physique d'une enveloppe rétrodiffusée supraluminique du type onde solitaire et on suggère l'observation d'une telle onde dans un laser SBS en anneau à fibre optique.

\section{INTRODUCTION}

In the latest advances of nonlinear optics, stimulated Brillouin backscattering (SBS) has received much attention mostly because it is the dominant stimulated scattering process in many optical media. Besides, as demonstrated in recent experiments ${ }^{1}$, it offers the possibility of achieving efficient pulse compression accompanied by a considerable power increase. Since the first observation of such a mechanism of compression in backward stimulated Raman scattering ${ }^{2}$, other authors ${ }^{3,4}$ have been confirming its high efficiency from a theoretical point of view. Anyhow, in practice, limitations always occur for such a compression, which may be due to the presence of damping or even to higher order nonlinear mechanisms such as the nonlinear Kerr effect.

Here, it is our aim to introduce some new developments in the comprehension of SBS under essentially nonstationary conditions. Within the frame of the standard description for the 3 wave resonant SBS interaction (obtained through the envelope approximation), we shall bring out several heuristic concepts in view of the experimental verification of typical dynamical behaviors arising in the formation and the amplification of the backscattered Stokes pulse. In the course of our investigation, we have been advantageously guided by the results obtained via numerical simulations of the process for various experimental conditions. Hence an important part of our arguments will rest upon interpretations of some of those results which bear particular significance to us.

From a theoretical point of view, it has been proved ${ }^{5}$ that the Inverse Scattering Transform (IST) is applicable to the problem of 3 -wave resonant interaction. However, the general scheme allowing connection between two solutions infinitely separated in time is not of a very practical use. Simplifications are readily obtained when one considers spatially bounded envelopes which are initially nonoverlapping ${ }^{6}$. In the case of SBS, no soliton exchange can take place because only the backscattered envelope may contain solitons. Besides, due to their spatial boundedness, the solutions to the 3 -wave problem investigated by the IST display no decay of radiation (here and later in this paper, the word "radiation" is used as opposed to "soliton"), thus preventing the observation of asymptotically well-shaped solitary pulses.

Before going into any further considerations, we think it important to outline the essential features of the SBS time evolution for a spatially bounded Stokes pulse in the presence of a counter- 
propagating constant pump input. In a first stage, its amplification strongly depends on the shape of its leading edge, but for long enough times it assumes the shape of a self-similar profile whose width decreases as the inverse of time while its leading maximum amplitude grows linearly in time 4. This asymptotic behavior seems to be quite irrespective of the presence of damping for the lowfrequency wave - i-e the sound wave for SBS - at least regarding the front part of the Stokes pulse. Clearly, the assumption of a finite support for the backscattered envelope is physically relevant when one envisages formation and amplification of the Stokes pulse over a finite interaction length.

The situation may drastically differ when the interaction length is infinite or, to be more precise, for spatially extended envelopes - this may be experimentally achieved, for instance, in a ring configuration. In this case, after each passage through the interaction medium, the Strokes pulse is re-injected (with a given coupling efficiency) at the other end of the medium, thus allowing for an infinite duration of the interaction. In this kind of configuration, one might envisage the possibility of an "induced dispersion" (using Chiu's expression ${ }^{7}$ ) due to the essentially nonlocal nature of the newly considered type of interaction, thus rendering radiation free to decay.

Aside from the IST approach to the 3-wave problem, specific solitary wave solutions were derived by Armstrong et al. ${ }^{8}$ and also discussed by Nozaki, Taniuti and Oshawa ${ }^{9}$ who were especially interested in the forward scattering case. In the next section, we shall introduce the corresponding solutions for the backscattering case and discuss some of their properties. In particular we shall a.ddress ourselves to these solitary wave solutions in which the three envelopes travel along with the same speed, this one being greater than the group velocities of all three waves in absolute value. On the basis of previous studies concerning the linear stability of such solutions, we shall argue in favor of their physical relevance in the frame of a ring geometry, supported by numerical results. We shall place ourselves in the realistic case of three damped waves, also allowing for a time modulation of the pump input. Typically, soliton-like profiles will be generated in the backscattered envelope, their essential features being very similar to those of the above cited solitary wave solutions.

Recently, it has been experimentally shown ${ }^{10}$ that a fairly good stabilization of an SBS-fiberring laser is achieved by a strong modulation of the pump input, synchronized with the round-trip period of the backscattered pulse (photon flight time associated to the length of the ring). Moreover, a good agreement is met between the experimental results and those obtained from the numerical simulation of the 3-wave resonant model. Motivated by these results, we suggest an experimental verification of the "superluminous amplification" of the Stokes pulse through a measurement of its recurrence time.

\section{THE COHERENT 3 - WAVE SBS EQUATIONS : ASYMPTOTIC SOLUTIONS}

The amplification and compression of a Stokes envelope $E_{2}$ counterpropagating with respect to a long duration pump wave $E_{1}$ in resonant SBS interaction $\left(\omega_{1}=\omega_{2}+\omega_{s} ; \mathbf{k}_{1}=\mathbf{k}_{2}+\mathbf{k}_{s} \Longleftrightarrow\right.$ $k_{s}=k_{1}+k_{2} \approx 2 k_{1}$ ) with a sound wave $E_{s}$, is described by the one-dimensional 3-wave equations (in dimensionless form) :

$$
\begin{gathered}
\left(\partial_{t}+\partial_{x}+\mu_{e}\right) E_{1}=-E_{2} E_{s} \\
\left(\partial_{t}-\partial_{x}+\mu_{e}\right) E_{2}=E_{1} E_{s}^{*} \\
\left(\partial_{t}+\epsilon \partial_{x}+\mu\right) E_{s}=E_{1} E_{2}^{*}
\end{gathered}
$$

where the group velocities $c / n$ of the E.M. envelopes $E_{1}$ and $E_{2}$ are both normalized to unity in absolute value, and $\epsilon$ stands for the ratio of the sound velocity to $c / n . \mu_{e}$ (resp. $\mu$ ) is the damping rate for the E.M. waves (resp. the acoustic wave).

Since we consider interaction in a homogeneous medium, when one of the amplitudes is initially zero, the choice of initial phases is arbitrary and their subsequent evolutions are trivial except for $\pi$ shifts related to a change in sign for one of the amplitudes. Hence, without lack of generality, we can choose all the envelopes to be real at any time $t \geq 0$.

In typical experiments in optical fibers we have $\epsilon \simeq 10^{-5}$ so that we choose to neglect the $\epsilon \partial_{\boldsymbol{x}}$ term in the last equation. Besides, in a first stage, we shall also neglect the damping terms in Eqs. (1), yielding 


$$
\begin{gathered}
\left(\partial_{t}+\partial_{x}\right) E_{1}=-E_{2} E_{s} \\
\left(\partial_{t}-\partial_{x}\right) E_{2}=E_{1} E_{s} \\
\partial_{t} E_{s}=E_{1} E_{2} .
\end{gathered}
$$

As demonstrated in Ref.4, an approximate self-similar asymptotic solution to Eqs.(2) can be derived for an initially bounded Stokes envelope $E_{2}$ counterpropagating with respect to a constant pump $E_{1}$. Indeed, using the change

$$
z=\frac{x+t}{2} \quad \tau=\frac{t-x}{2}
$$

the SBS equations (2) become :

$$
\begin{gathered}
\partial_{z} E_{1}=-E_{2} E_{s} \\
\partial_{\tau} E_{2}=E_{1} E_{s} \\
\frac{1}{2}\left(\partial_{z}+\partial_{\tau}\right) E_{s}=E_{1} E_{2} .
\end{gathered}
$$

Now, as stated in Ref.3, for a sufficiently large amplitude of the leading $E_{2}$ peak $\left(E_{2 \max }>1\right)$, the dependence on $\tau$ of all the envelope amplitudes near this leading peak can be neglected in comparison to their dependence on $z$, thus rendering system (4) analogous to the system of equations describing the propagation of a pulse in an inverted two-level medium, known as the self-induced transparency effect ${ }^{11,12}$ :

$$
\begin{gathered}
\partial_{z} E_{1}=-E_{2} E_{s} \\
\partial_{\tau} E_{2}=E_{1} E_{s} \\
\frac{1}{2} \partial_{z} E_{s}=E_{1} E_{2} .
\end{gathered}
$$

Within rescaling of both variables and fields, system (5) also describes the transient stimulated Raman (forward) scattering as introduced in Ref.13 and assumed to modelize the experimental observations in Ref.14. In Raman scattering, $E_{1}$ and $E_{s}$ would correspond to the envelopes of the pump and Stokes fields and $E_{2}$ to the induced polarization density of the material medium $\left(\omega_{1}=\omega_{2}+\omega_{s} ; \mathbf{k}_{1}=\mathbf{k}_{2}+\mathbf{k}_{s} \Longleftrightarrow k_{1}=k_{2}+k_{s} \simeq k_{s}\right)$.

Defining the self-similar variable $\xi=z \tau$ and writing the fields as :

$$
\begin{gathered}
E_{1}(z, \tau)=\cos [\phi(\xi) / 2] \\
E_{2}(z, \tau)=\frac{\tau}{\sqrt{2}} \frac{d \phi}{d \xi} \\
E_{s}(z, \tau)=\sqrt{2} \sin [\phi(\xi) / 2],
\end{gathered}
$$

yields the following ordinary differential equation :

$$
\xi \frac{d^{2} \phi}{d \xi^{2}}+\frac{d \phi}{d \xi}=\sin \phi
$$

One can easily check that $\partial_{\tau} E_{s} / \partial_{z} E_{s}=z / \tau$. Hence, at fixed $z \neq 0$, one can effectively neglect $\partial_{\tau} E_{s}$ in the lhs of the last equation of system (4) as $\tau$ tends to infinity.

The self-similar asymptotic solution (here called " $\pi$-pulse" after Lamb ${ }^{11}$ ) obtained by solving Eqs. $(6 a, b)$, is shown in Fig.1 as a function of $\xi$ for a given large value of $r$. Actually, the validity of approximating Eqs.(4) by Eqs.(5) is restricted to a domain of the $z$-axis which does not include the leading edge point $z_{0}$ of $E_{2}$ [i.e. $E_{2}\left(z<z_{0}\right)=0$ with $E_{2}$ continuous at $z_{0}$ ]. However, as noted in Ref.4 and analogously emphasized in Ref.15, it proves to be a "continuation" in the nonlinear region of the linearized version of Eqs.(4). Indeed, equating $E_{1}$ to unity, the asymptotic form of the solution $E_{2}$ for the linearized problem equally displays self-similarity in the variable $\xi$ (see Appendix C in Ref.4). 
Before considering other types of solution, one should remark that, on the infinite line, the growing of a self-similar asymptotic solution of the $\pi$-pulse type occurs for any spatially bounded initial profile of the backscattered envelope against a constant pump input (even when allowing for damping of the sound wave).

Next, we discuss the solitary wave solutions (travelling waves) which are special cases of a broad class of soliton solutions fully described by Chiu (see Ref.7 and references therein). Wishing to avoid the rather lengthy calculations involved in the IST approach, we shall use the more heuristic one formerly used by Armstrong et al. ${ }^{8}$ and by Nozaki et al. ${ }^{9}$. yields :

Starting from Eqs.(2), we first perform the change of frame $: x \longrightarrow x+v t, t \rightarrow t$ which

$$
\begin{gathered}
{\left[\partial_{t}+(1+v) \partial_{x}\right] E_{1}=-E_{2} E_{s}} \\
{\left[\partial_{t}+(v-1) \partial_{x}\right] E_{2}=E_{1} E_{s}} \\
{\left[\partial_{t}+v \partial_{x}\right] E_{s}=E_{1} E_{2} .}
\end{gathered}
$$

Then, by defining the $A_{i}$ 's fields as

$$
\begin{gathered}
A_{1}=|1+v|^{1 / 2} E_{1} \\
A_{2}=|v-1|^{1 / 2} E_{2} \\
A_{3}=|v|^{1 / 2} E_{s}
\end{gathered}
$$

and looking for stationary solutions in the new frame, we have :

$$
\begin{gathered}
\partial_{X} A_{1}=-s_{1} A_{2} A_{3} \\
\partial_{X} A_{2}=s_{2} A_{1} A_{3} \\
\partial_{X} A_{3}=s_{3} A_{1} A_{2}
\end{gathered}
$$

where $X=x /|(v-1)(1+v) v|^{1 / 2}$ and $s_{1}=\operatorname{sgn}(1+v), s_{2}=\operatorname{sgn}(v-1), s_{3}=\operatorname{sgn}(v)$. The only possibility for $A_{1}$ to be assigned the rôle of the pump [i-e $A_{1}(x=-\infty)=$ const. and $A_{2,3}(x= \pm \infty)=0$ ], is that $\left(s_{1}, s_{2}, s_{3}\right)= \pm(1,1,1)$ or equivalently $|v|>1$ (cf. Ref.16). Taking arguments of stability given in Ref.7 into account, we shall be concerned only with the solutions for which $v>1$ (the $v<-1$ case only intervenes in a transient stage of the nonlinear interaction and is asympiotically unstable). The most general solutions to Eqs.(9) involve Jacobi elliptic functions but if we restrict ourselves to infinite period solutions, they read:

$$
\begin{gathered}
A_{1}=\mp A \tanh A X \\
A_{2}= \pm A_{3}=A \operatorname{sech} A X .
\end{gathered}
$$

This triple solitary waves solution is shown in the original variables in Fig.2. Figs.3(a,b,c) show the counterpropagation of an initial profile for $E_{2}$ given by (10) with $A=(1+v)^{1 / 2}$ and $v=$ 1.01 , with respect to a constant pump $E_{1}=1$ and a vanishing initial sound amplitude $E_{s}=0$. For long interaction times, a "superluminous" $(v=1.01)$ triple solitary waves structure of the form given in Eqs.(10) leaves the radiative envelope ( $\pi$-pulse structure) which moves at light velocity $(v=1)$. For longer times $(t \simeq 85)$ a second solitary structure appears. However, this "superluminous" generation of solitary waves will become impeded as soon as the $E_{2}$ background in front of its leading edge gets vanishingly small. Then, the self-similar amplification, which causes a "superluminous" displacement, can no longer take place and causality imposes a further evolution of the $\pi$-pulse type. Figs.4(a,b) show the transformation of the "superluminous" quasi-soliton into the "luminous" $\pi$-pulse envelope for $v=1.02$. We stress the importance of an extended $E_{2}$ background for a self-similar "superluminous" amplification of the solitary type to occur. This can be obtained via a ring configuration where the leading $E_{2}$ pulse propagates over a permanently excited $E_{2}$ background. 


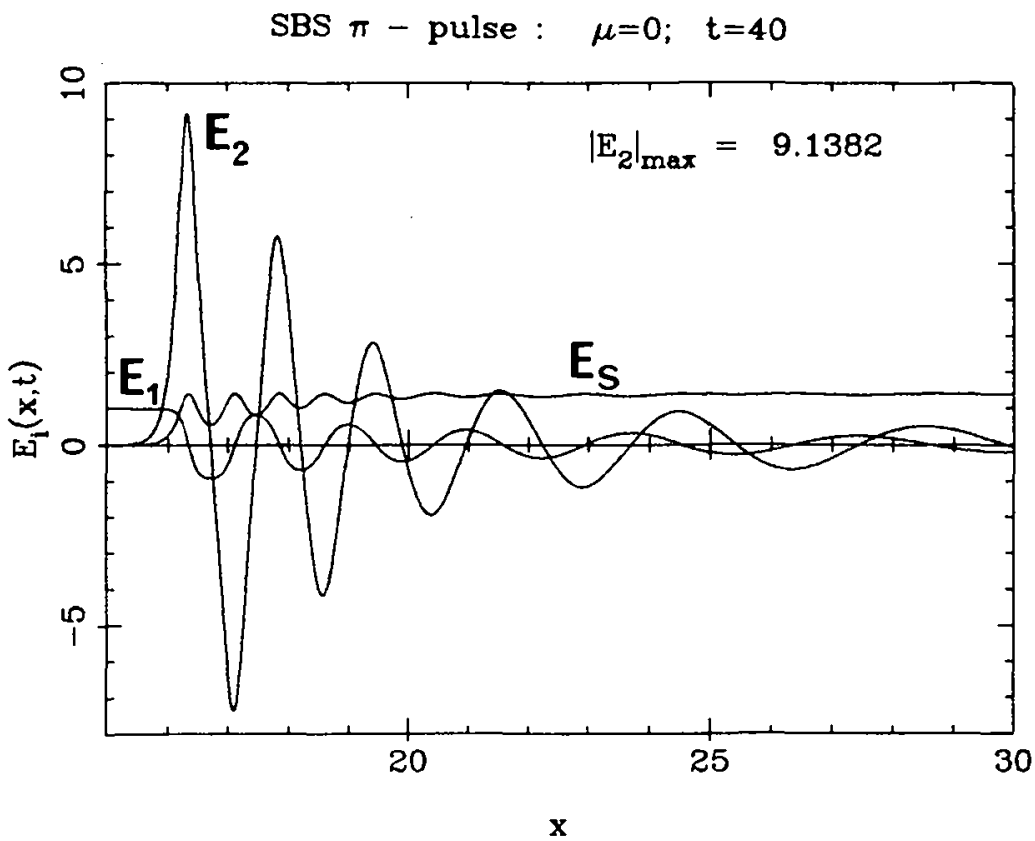

Fig.1 Amplitude spatial distribution for the $\pi$-pulse in the frame of the backscattered envelope.

SBS sol.:

$$
\mu=0 ; \quad t=65
$$

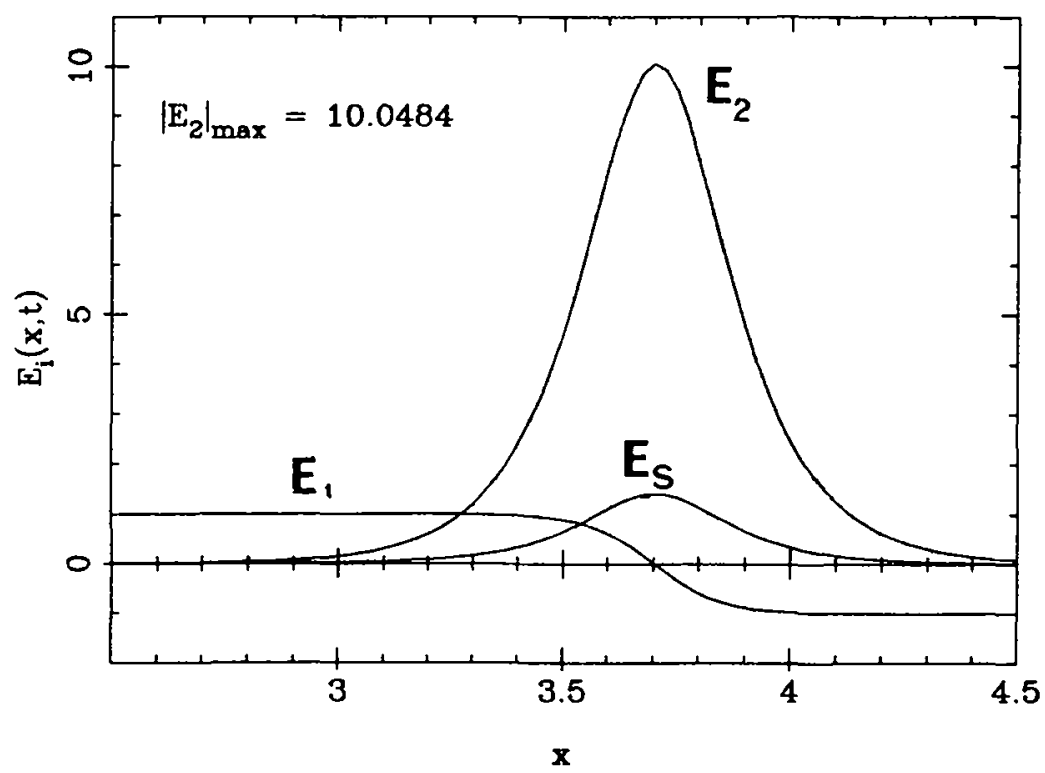

Fig.2 Triple solitary waves solution given by formulas $(8,10)$ for $v=1.01$ and $A=(1+v)^{1 / 2}$.

\section{GENERATION OF SUPERLUMINOUS STOKES PULSES IN A REAL FIBER-RING CONFIGURATION}

First of all, in a real optical experiment, dissipation cannot be neglected. However, it is of little consequence for the type of asymptotic solutions presented above. Sound damping does 
not affect amplification nor compression of the leading backscattered Stokes pulse in the $\pi$-pulse evolution (see Ref.4). Moreover, dissipation plays a stabilizing rôle in the solitary wave evolution, imposing maximum amplitude and velocity and smearing out the leaving radiative tail. Numerical simulations in the presence of dissipation $\left(\mu_{e} \neq 0, \mu \neq 0\right)$ display a high stability of the quasi-soliton solution.

Now the problem is to generate the "superluminous" envelopes in a real experiment.

Here we shall present numerical results corresponding to a parallel experiment ${ }^{10}$ performed with an optical fiber-ring cavity of length $L=83 \mathrm{~m}$, pumped by a single mode Ar-ion CW laser beam of wavelength $\lambda=5145 \AA$. A stable train of SBS Stokes pulses, compressed to $10 \mathrm{~ns}$, has been obtained by strong modulation of the laser beam. Interruption of the pump action at each round-trip time $t_{r}=L n / c \simeq 415 n s$, allows spontaneous damping of the excited acoustic waves which otherwise accumulate at the input end of the fiber and shorten the effective length of the nonlinear interaction medium by depleting the pump just at its entry. Pump modulation is therefore a good mechanism to use the maximum fiber depth. In Ref.10, the modulation is introduced through an intra-cavity acousto-optic modulator which modulates not only the pump but also the backscattered Stokes envelope and therefore reduces its background width. The train of Stokes pulses presents the round-trip period $t_{r}$ corresponding to a propagation at the light velocity $c / n$ in the fiber : $\pi$-pulse behavior.

Now, if we only modulate the pump and recouple the whole Stokes envelope, we allow the Stokes background to fill the total length of the fiber. In Figs.5(a,b) we present the results of a numerical simulation of Eqs.(1) for the ring configuration in the latter case. The dimensionless length $L=40$ corresponds to a maximum input pump power of $25 \mathrm{~mW}$ in a silica fiber of $83 \mathrm{~m}$ length and $3 \mu m$ core diameter. The characteristic SBS gain length is about $2 m$ and the feedback efficiency $\rho \equiv\left|E_{2}(x=L, t) / E_{2}(x=0, t)\right|^{2} \simeq 0.56$. Fig.5a shows the time evolution of the backscattered envelope $\left|E_{2}(x=0, t)\right|$ versus time. After a transient amplification stage, an almost periodic regime of Stokes pulses sets in, where the leading edges are separated by pseudo-periods lower than $t_{r}$ (cf. Fig.5b), clearly displaying the "superluminous" behavior of the solitary wave type. Figs.6 show the spatial distribution of the envelope amplitudes inside the fiber at consecutive periods, before and after the amplitude saturation shown in Fig.5a. One can directly see the generation of the quasi-soliton structure of $E_{2}$ as a self-similar amplification of the Stokes background.

${ }^{1}$ D.T. Hon, Opt. Lett. 5, $516(1980)$

${ }^{2}$ W. Maier, W. Kaiser, and J.A. Giordmaine, Phys. Rev. Lett. 17, 1275 (1966)

${ }^{3}$ V.A. Gorbunov, Sov. J. Quant. Electron. 14, 1066 (1984)

${ }^{4}$ J. Coste and C. Montes, Phys. Rev. A 34, 3940 (1986)

${ }^{5}$ V.E. Zakharov and S.V. Manakov, JETP Lett. 18, 243 (1973); Sov. Phys. JETP 42, $842(1976)$

${ }^{6}$ D.J. Kaup, Stud. Appl. Math. 55, 9 (1976);

D.J. Kaup, A. Reiman, and A. Bers, Rev. Mod. Phys. 51, 275 (1979)

${ }^{7}$ S.C. Chiu, J. Math. Phys. 19, 168 (1978)

8 J.A. Armstrong, S.J. Sudhanshu, and N.S. Shiren, J. Quant. Elect. QE-6, 591 (1974)

${ }^{9} \mathrm{~K}$. Nozaki and T. Taniuti, J. Phys. Soc. Jpn. 34, 796 (1973);

Y. Oshawa and K. Nozaki, J. Phys. Soc. Jpn. 36, 591 (1974)

10 J. Botineau, C. Leycuras, C. Montes, and E. Picholle, "Stabilization of stimulated

Brillouin fiber ring laser by strong pump modulation", J.O.S.A. B to be published

11 G.L. Lamb, Jr., Phys. Lett. 29 A, 507 (1969); Rev. Mod. Phys. 43, 99 (1971)

12 V.E. Zakharov, JETP Lett. 32, 589 (1980)

${ }^{13}$ F.Y.F. Chu, and A.C. Scott, Phys. Rev. A, 12, 2060 (1975)

${ }^{14}$ K. Drühl, R.G. Wenzel, and J.L. Carlsten, Phys. Rev. Lett. 51, 1171 (1983)

${ }^{15}$ I.R. Gabilov, V.E. Zakharov and A.V. Mikhailov, JETP Lett. 37, 279 (1983)

${ }^{16}$ C.J. McKinstrie, Phys. Fluids 31, 288 (1988) 

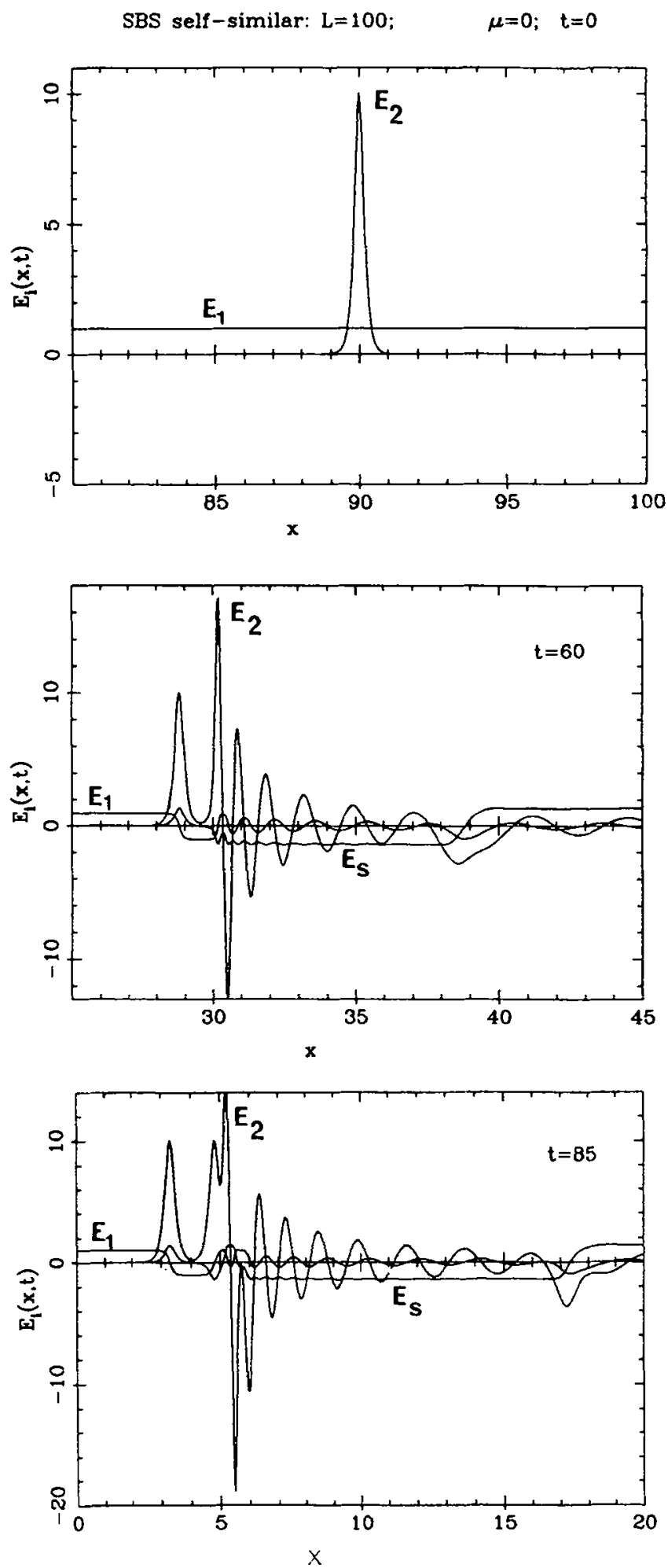

Figs.3 Time evolution of an initial Stokes envelope of the hyperbolic secant type $(t=0)$. At $t=60$, a triple solitary structure is well separated from the radiative $\pi$-pulse tail. At $t=85$, a second solitary structure leaves the tail. 

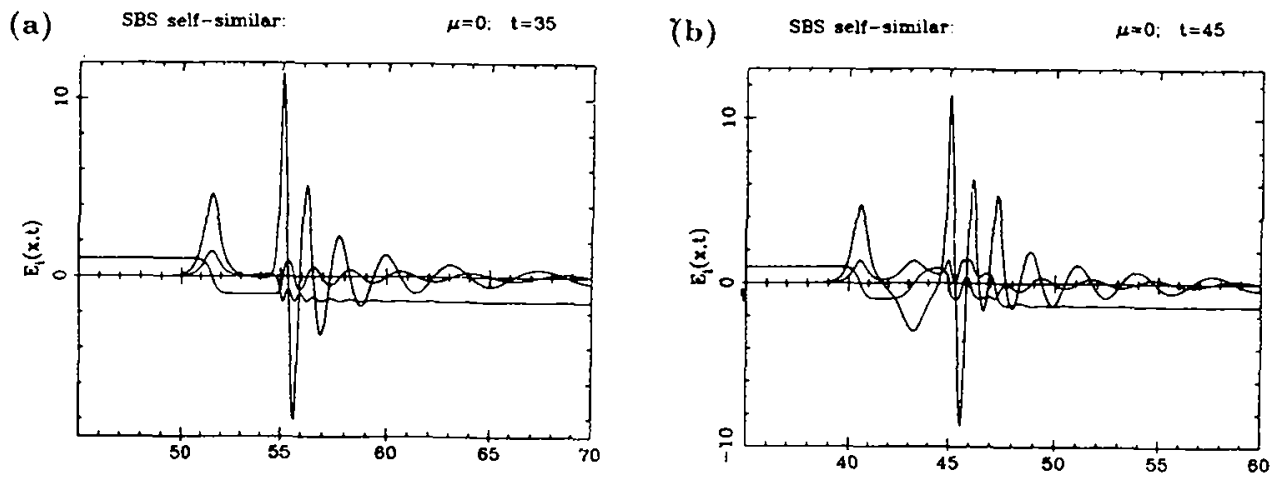

Figs.4 Transformation of the solitary wave structure moving at "superluminous" velocity $v=1.02$ (a) into the $\pi$-pulse structure moving at light velocity $v=1$ (b).

$$
\text { SBS laser-ring: } \mathrm{L}=40 ; \rho=0.56 ; \quad \mu=5 ; \mu_{\mathrm{e}}=5 \times 10^{-3}
$$
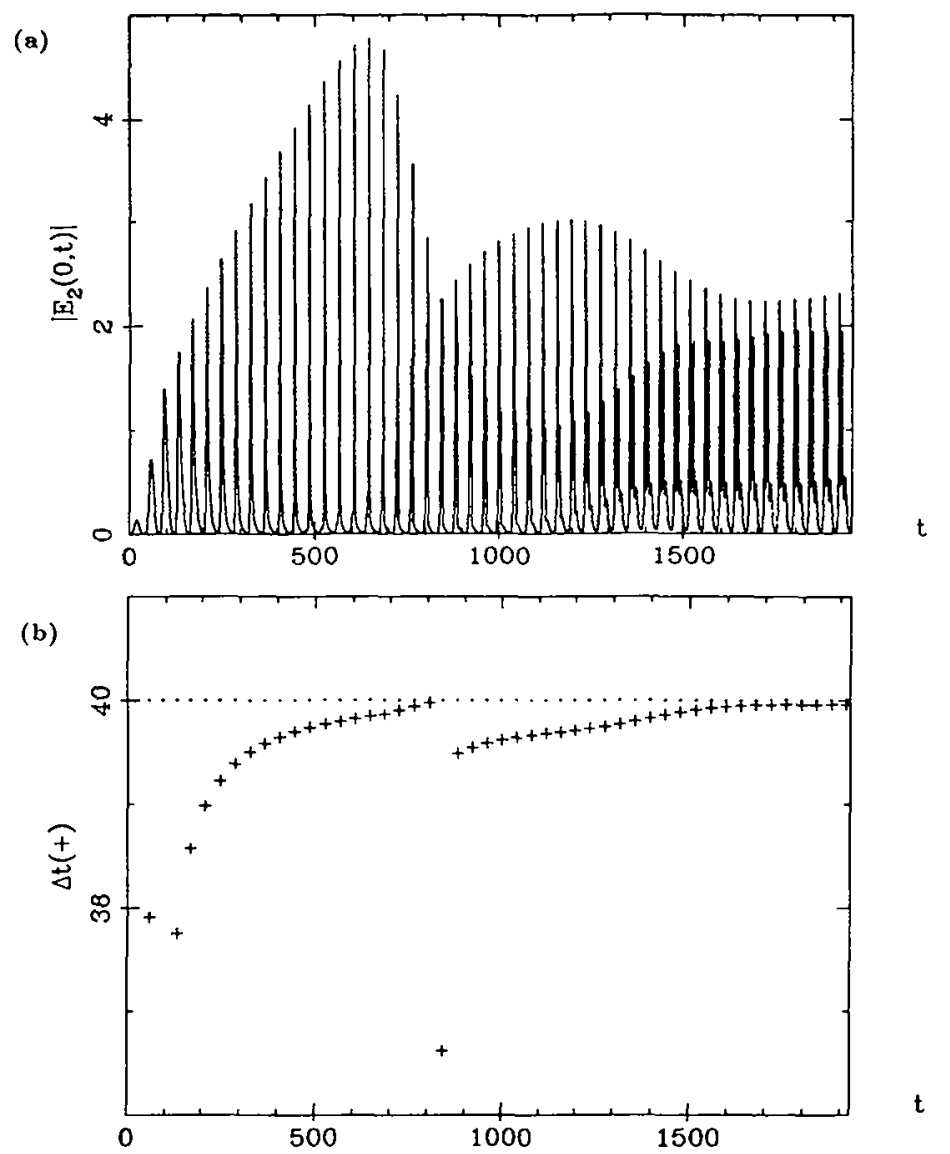

Figs.5 (a) Time evolution of the backscattered amplitude $\left|E_{2}(x=0, t)\right|$ in the ring configuration, where only the pump is modulated, obtained from Eqs.(2).

(b) Time intervals between two pulses (pseudo-periods) versus time. $\Delta t<40$ represent "superluminous" pseudo-periods, since $\Delta t=40$ is the round-trip period (or photon flight time associated to the length $L=40$ of the ring). 
SBS ring: $L=40 ; \rho=0.56 ; \mu=5 ; \mu_{\mathrm{e}}=5 \times 10^{-3}$
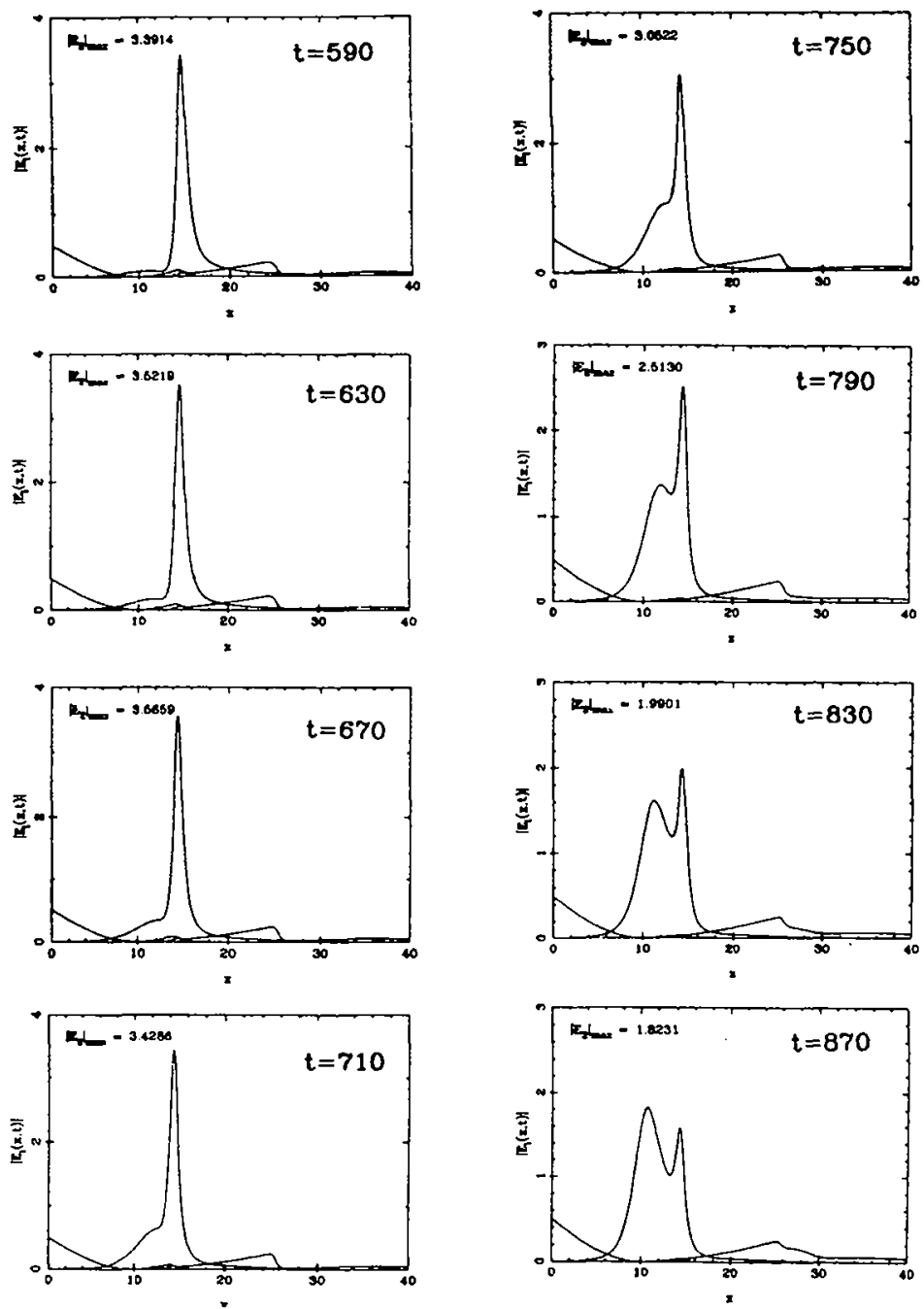

Fig.6 Time sequence of amplitude spatial distribution. Generation of the quasisoliton structure for $E_{2}$ as a self-similar amplification of the Stokes background. From $t \simeq 800$ on the sech function [cf. formula(10)] fits very well the leading profile of the Stokes pulse. 